\title{
A evolução do registro e prescrição de fitoterápicos no Brasil sob a perspectiva legal e sanitária
}

\author{
The evolution in the registration and prescription of herbal \\ medicines in Brazil from legal and sanitary perspectives
}

\author{
Mariana Cardoso Oshiro* \\ Marilis Dallarmi Miguel \\ Josiane de Fátima Gaspari Dias \\ Eliane Carneiro Gomes \\ Obdulio Gomes Miguel
}

Universidade Federal do Paraná (UFPR), Curitiba, PR, Brasil

*E-mail: marianac.oshiro@gmail.com

\section{RESUMO}

Este artigo propõe-se a avaliar a regulamentação de registro de fitoterápico em vigor (RDC $\mathrm{n}^{\circ} 26$, de 13 de maio de 2014) e as perspectivas em relação à prescrição de fitoterápicos no Sistema Único de Saúde (SUS). Para tanto, analisou-se o histórico das medidas políticas relacionadas à introdução de fitoterápicos no sistema de saúde brasileiro e as legislações de registro de fitoterápicos que vigoravam anteriormente à publicação da RDC n 26/14. A classificação dos fitoterápicos elaborada pela legislação em vigor (medicamento fitoterápico e produto tradicional fitoterápico) permitiu a visualização imediata da comprovação de segurança e eficácia do medicamento, uma vez que nem todas as plantas medicinais possuem estudos clínicos para comprovação de sua segurança e eficácia. A nova legislação e as monografias de fitoterápicos apresentados na Farmacopeia Brasileira trouxeram maior credibilidade e segurança aos prescritores e profissionais de saúde com relação ao tratamento medicamentoso com fitoterápicos. A atual legislação de registro de fitoterápicos revela a constante preocupação das autoridades brasileiras em desenvolver normas que corroborem com a Política Nacional de Práticas Integrativas e Complementares (PNPIC) e com a Política Nacional de Plantas Medicinais e Fitoterápicos (PNPMF) introduzidas no SUS e as recomendações da Organização Mundial de Saúde (OMS) em promover qualidade no estado de saúde da população brasileira.

PALAVRAS-CHAVE: Saúde Pública; Fitoterapia; Regulamentação de Organismos de Política

\section{ABSTRACT}

The aim of this paper is to review the herbal registration regulations (RDC no. 26/14) and to evaluate the outlook for prescription of herbal medicine at SUS. For this purpose, we've tracked and analyzed the record of political measures related to herbal medicine within the Brazilian health system as well as herbal registration norms which existed prior to the publication of RDC no. 26/14. The classification of herbal medicine formulated by legislation (herbal medicine and traditional herbal product) allows immediate visualization of safety and efficacy, since not all medicinal plants have clinical studies to prove safety and efficacy. The new legislation and herbal medicine monographs presented in Brazilian Pharmacopeia have brought reliability and safety to prescribers and healthcare professionals regarding drug treatment through herbal medicine. The current herbal registration legislation reveals the unceasing concern from Brazilian authorities to develop legislation that corroborates with the National Policy on Integrative and Complementary Practices and with the National Policy of Herbal Medicine at SUS and in agreement with World Health Organization recommendations in order to foster the quality of health among the Brazilian population.

KEYWORDS: Public Health; Phytotherapy; Regulations for Policy Organizations 


\section{INTRODUÇÃO}

No Brasil, a fitoterapia foi institucionalizada nacionalmente no Sistema de Saúde Pública (SUS) com a publicação da Política Nacional de Práticas Integrativas e Complementares (PNPIC) em 2006, documento norteador do desenvolvimento da Política Nacional de Plantas Medicinais e Fitoterápicos. Desde sua implementação, novas diretrizes para inserção e regulamentação da utilização de fitoterápicos no SUS veem sendo desenvolvidas. As políticas instituídas são importantes para possibilitar o acesso de toda a população brasileira às práticas fitoterápicas e a padronização da mesma. Ainda assim, algumas espécies vegetais não possuem estudos clínicos para comprovação de segurança e eficácia. Neste contexto, a Resolução $n^{\circ} 26$, de 13 de maio de 2014', que dispõe sobre o registro de medicamentos fitoterápico e o registro/notificação de produto tradicional fitoterápico, reforça o tripé segurança, eficácia e qualidade dos fitoterápicos, no qual a PNPIC se sustenta. Este artigo tem como objetivo avaliar a regulamentação vigente no que se refere ao registro de fitoterápicos e às perspectivas em relação à prescrição de fitoterápicos no SUS.

\section{MÉTODO}

Trata-se de uma pesquisa qualitativa em bases de dados e sites oficiais tais como Agência Nacional de Vigilância Sanitária (Anvisa) e Diário Oficial da União (DOU), no período de 1967 até 2016, utilizando como descritores as palavras: fitoterápicos, prescrição, plantas medicinais, diretrizes, Política Nacional de Práticas Integrativas e Complementares (PNPIC) e Política Nacional de Plantas Medicinais e Fitoterápicos (PNPMF). Foram selecionadas e analisadas as seguintes legislações que regulamentam o registro de fitoterápico no Brasil: Portaria $n^{\circ} 22$, 30 de outubro de 1967; Portaria $n^{\circ} 06$, de 31 de janeiro de 1995; Resolução $n^{\circ} 17$, de 24 de fevereiro de 2000; Resolução $n^{\circ} 48$, de 16 de março de 2004; Resolução $n^{\circ} 14$, de 31 de março de 2010 e Resolução $n^{\circ} 26$, de 13 de maio de 2014. Neste mesmo período, analisaram-se documentos publicados por órgão federal referente à prescrição de fitoterápicos no SUS, como a PNPIC, a PNPMF e a Resolução RDC $n^{\circ}$ 84, de 15 de junho de 2016. Assim como a Resolução RDC n 60, de 10 de novembro de 2011, documento publicado por órgão federal referente às práticas de manipulação e dispensação de fitoterápicos.

Adicionalmente, no mesmo período de tempo, foi realizado um levantamento bibliográfico referente ao histórico da fitoterapia em sites de busca como SciELO e Science Direct, utilizando os descritores: fitoterapia, legislação, plantas medicinais e saúde pública. O estabelecimento de um plano de fundo histórico com os acontecimentos mundiais e nacionais referente ao uso de fitoterápicos favoreceu a análise das legislações e documentação selecionadas e permitiu construir um cenário nacional da inserção dos fitoterápicos no Sistema de Saúde Pública. Dessa forma, possibilitou a discussão das mudanças, avanços e medidas para a regulamentação e prescrição de fitoterápicos no SUS.

\section{RESULTADOS}

\section{Fitoterapia na Saúde Pública}

No ano de 1976, a Organização Mundial de Saúde (OMS) realizou a $29^{\mathrm{a}}$ Assembleia Mundial de Saúde no intuito de promover a igualdade no estado de saúde dos povos do mundo. A OMS reconheceu o valor potencial da medicina tradicional para a expansão dos serviços básicos de saúde à população, principalmente em regiões onde a medicina moderna não conseguia atuar. Posteriormente, foram publicadas as resoluções WHA 29.72 (1976) e WHA 30.49 (1977) que solicitaram aos países-membros o desenvolvimento de políticas nacionais para a integração da medicina tradicional no sistema de saúde de cada país ${ }^{2,3}$. Em 1978, foi publicada a redação Alma-Ata ${ }^{4}$ realizada na Conferência Internacional de Cuidados Primários em Saúde, evento que alertou os governos do mundo para a desigualdade de saúde entre os povos, principalmente nos países em desenvolvimento, e incentivou o acesso aos serviços de saúde que garantem qualidade de vida e bons níveis de saúde. Neste mesmo ano, a OMS reconheceu a importância da utilização de plantas medicinais no sistema de saúde e, por meio da resolução WHA 31.33 (1978), sugeriu a padronização da nomenclatura botânica, a classificação terapêutica e a revisão de dados científicos sobre a eficácia terapêutica das plantas medicinais ${ }^{5}$. Tendo em vista os critérios que garantem a qualidade dos produtos fitoterápicos comercializados atualmente, a OMS, em 1987, por meio da resolução WHA 40.33 (1987), enfatizou a necessidade de iniciar programas relativos à identificação, cultivo e conservação de espécies vegetais utilizadas na medicina tradicional. E ainda exigiu o controle de qualidade das drogas vegetais através de técnicas modernas e boas práticas de industrialização na produção de medicamentos tradicionais ${ }^{6}$.

Apesar dos esforços da OMS em introduzir a utilização de plantas medicinais na assistência à saúde, em muitos países a medicina tradicional ainda não tinha sido adotada como política. No intuito de auxiliar os países-membros a padronizar as metodologias de pesquisa nesta área, a OMS, em 1991, publicou guias técnicos para avaliação de segurança e eficácia de plantas medicinais (Guidelines for the assessment of herbal medicines e Research guidelines for evaluating the safety and efficacy of herbal medicines) ${ }^{7}$. Não sendo suficientemente abrangente para cobrir todos os aspectos relacionados à pesquisa de plantas medicinais, em 2002, a OMS publicou, após longo período de debates com especialistas e autoridades de saúde do mundo, um guia estratégico de políticas públicas para o uso racional e integrado da medicina tradicional e medicina complementar/alternativa (WHO Traditional Medicine Strategy 2002-2005 e, mais recentemente, o WHO Traditional Medicine Strategy 2014-2023) tendo como objetivos principais a construção de conhecimento base para formulação de políticas nacionais, legislações que garantem segurança, qualidade e eficácia de medicamentos tradicionais e a integração da medicina tradicional no sistema de saúde ${ }^{8}$. 
No Brasil, em consonância com as políticas da OMS, foi adotada, em 2006, a PNPIC no SUS. A publicação da PNPIC institucionalizou no SUS não só os serviços que envolvem plantas medicinais e fitoterapia, mas serviços como: homeopatia, medicina tradicional chinesa/acupuntura, medicina antroposófica e termalismo social, como práticas integrativas e complementares, permitindo o acesso da população à fitoterapia e as demais práticas consideradas alternativas ao tratamento de doenças'. Anteriormente à publicação da PNPIC, alguns municípios do país já desenvolviam projetos relacionados à introdução da fitoterapia na rede de saúde pública municipal. Entretanto, devido à ausência de uma política nacional, os projetos de saúde ocorreram de maneira desigual e descontínua em diferentes regiões do Brasil, sem o devido acompanhamento da saúde do paciente e ausência de controle de qualidade dos insumos fornecidos para a produção de fitoterápicos.

As discussões mundiais sobre a integração da medicina tradicional no sistema de saúde pública, iniciada pela OMS, serviram como referência para elaboração de documentos e realização de conferência em âmbito nacional. Dentre os documentos relativos ao assunto podemos destacar:

- Resolução ${ }^{\circ} 08$, de 08 de março de $1988^{10}$, elaborada pela Comissão Interministerial de Planejamento e Coordenação (Ciplan). Previa a criação de procedimentos e rotinas relativos à prática da fitoterapia nas unidades de assistência médica, criação, em cada Estado, de um grupo de trabalho para estudar, planejar e coordenar a implantação do programa de fitoterápicos e previa a normatização da área e treinamento de recursos humanos.

- Relatório da $10^{\mathrm{a}}$ Conferência Nacional de Saúde ${ }^{11}$, realizada entre os dias 2 e 6 de setembro de 1996, em Brasília-DF, que apresentou uma Proposta de Política Nacional de Assistência Farmacêutica integrada aos princípios do SUS. Como um dos deveres desta política, podemos destacar o incentivo à fitoterapia e à homeopatia na assistência farmacêutica pública, assim como a garantia da universalização do acesso aos medicamentos e ações de vigilância farmacológica para medicamentos e plantas medicinais, reafirmando a necessidade de normas para incorporação e utilização da fitoterapia no SUS.

- Portaria $n^{\circ} 3.916$, de 30 de outubro de $1998^{12}$, que aprovou a Política Nacional de Medicamentos entre as diretrizes de desenvolvimento científico e tecnológico, ressalta o apoio à pesquisa que vise o aproveitamento do potencial terapêutico da flora e fauna nacionais, além da criação da Relação de medicamentos essenciais (Rename) como instrumento para assegurar o uso racional de medicamentos, através da padronização da terapêutica de doenças garantindo a integralidade do tratamento medicamentoso.

- Relatório do Seminário Nacional de Plantas Medicinais, Fitoterápicos e Assistência Farmacêutica ${ }^{13}$, realizado em 2003, que reforçou a recomendação de integrar o uso de plantas medicinais e medicamentos fitoterápicos no SUS.

- Relatório da $12^{\mathrm{a}}$ Conferência Nacional de Saúde ${ }^{14}$, realizada em 2003, que reiterou as recomendações da Conferência
Nacional de Medicamentos e incentivou o desenvolvimento da tecnologia de produção de medicamentos da flora brasileira, favorecendo a produção nacional e a implantação de programas para o uso de medicamentos fitoterápicos nos serviços de saúde.

- Resolução nº 338/04 do Conselho Nacional de Saúde ${ }^{15}$, que aprovou a Política Nacional de Assistência Farmacêutica e as ações intersetoriais para a utilização de plantas medicinais com respeito aos conhecimentos tradicionais incorporados com embasamento científico, qualificação e fixação de produtores, envolvimento dos profissionais de saúde no processo de incorporação dessa opção terapêutica e incentivo à produção nacional com a utilização da biodiversidade existente no país.

Em 2006, após publicação da PNPIC, foi lançada a PNPMF ${ }^{16}$. Esta política incentivou a criação de programas, projetos e atividades que colocassem em prática as experiências da fitoterapia, já desenvolvidas na rede pública de muitos municípios e Estados brasileiros, visando garantir o acesso seguro e uso racional de plantas medicinais e fitoterápicos em todo país, o desenvolvimento de tecnologias e inovações, assim como o fortalecimento da cadeia produtiva de fitoterápicos (cultivo, manipulação, fabricação e comercialização), por meio do uso sustentável da biodiversidade brasileira.

Como exemplo de ação que exemplifica esta política, podemos citar a criação da Relação Nacional de Plantas Medicinais de Interesse ao SUS (Renisus) que promoveu a inserção dos fitoterápicos no SUS em consonância com a Política Nacional de Saúde, Política Nacional de Assistência Farmacêutica e a PNPIC no SUS. Esta lista se baseou em espécies vegetais já utilizadas nos serviços de saúde estaduais e municipais, espécies vegetais que possuem comprovação de eficácia por meio de estudos etnofarmacológicos e estudos científicos podendo gerar produtos de interesse ao SUS ${ }^{17,18}$. Entretanto, de acordo com Torres (2009), um dos desafios em se incluir mais fitoterápicos no SUS seria a dificuldade no atendimento dos critérios regulatórios estabelecidos pela Anvisa ${ }^{19}$.

\section{Registro de fitoterápico no Brasil}

A comercialização de produtos fitoterápicos é regulada pela Anvisa. Para receber autorização de venda de produtos fitoterápicos, é necessária a apresentação de um dossiê contendo documentos e informações requeridos pela Anvisa (controle de qualidade, boas práticas de fabricação, validação de métodos analíticos, estudo de estabilidade, entre outros), que avalia e aprova a liberação do fitoterápico no mercado. A seguir, estão descritos aspectos relacionados à comprovação de segurança e eficácia encontrados nas legislações publicadas referentes ao registro de fitoterápicos no Brasil.

A Portaria $\mathrm{n}^{\circ} 22$, de 30 de outubro de $1967^{20}$, deu início à normatização do registro dos fitoterápicos. Para a realização do registro eram necessárias as informações sobre a identificação botânica, caracterização da droga vegetal, características 
organolépticas, características físico-químicas, fitoquímicas, fundamentação para o emprego terapêutico (referência ou trabalho científico), ausência de efeitos tóxicos e experimentação farmacológica em animal.

A primeira atualização da legislação para registro de fitoterápicos ocorreu em 1995, com a Portaria n ${ }^{\circ}$, de 31 de janeiro de $1995^{21}$, que introduziu, para comprovação de eficácia e segurança, a apresentação de estudos de toxicologia e farmacologia pré-clínica e clínica. Previa também a isenção de registro para os fitoterápicos que estivessem incluídos em monografias farmacopeicas ou códigos oficiais, desde que apresentadas informações referentes à ausência de toxicidade, indicações terapêuticas e controle de qualidade. A isenção do registro era concedida por meio do cadastramento do fitoterápico junto ao Ministério da Saúde ${ }^{22}$. Entretanto, antes do período estipulado para apresentação dos referidos estudos, a comunidade científica, juntamente com os órgãos reguladores, lançaram a Resolução $\mathrm{n}^{\circ} 17$, de 24 de fevereiro de $2000^{23}$, demonstrando haver empenho na regularização de fitoterápicos tanto do ponto de vista técnico-científico quanto legal.

A Resolução $n^{\circ}$ 17/2000 incluiu critérios para a comprovação de segurança e eficácia dos fitoterápicos. Adicionalmente à comprovação por meio de estudos clínicos e farmacológicos, a legislação trouxe o conceito de medicamento (fitoterápico) tradicional, no qual a comprovação de segurança e eficácia poderia ser feita através de levantamentos bibliográficos que comprovassem a utilização segura por um período igual ou superior a dez anos. Essa comprovação também poderia ser obtida por levantamentos literários a partir de uma lista de referências bibliográficas e um sistema de pontuação dos periódicos disponibilizada no Anexo II da Resolução. 0 documento disponibilizou também uma lista de referência de fitoterápicos (Anexo I), que poderiam ser registrados desde que respeitadas as especificações contidas, como exemplo: parte usada, formas de uso, indicações terapêuticas, dose e via de administração. Em complemento, inseriu a necessidade do teste de estabilidade para comprovação da validade do produto acabado.

A Resolução $n^{\circ} 48$, de 16 de março de $2004^{24}$, pouco diferiu de sua antecessora. Com relação à comprovação de segurança e eficácia, aumentou de 10 para 20 anos a comprovação da utilização segura através de levantamentos bibliográficos. E denominou por "registro simplificado de fitoterápicos" aqueles que seguissem a lista de referência de fitoterápicos presente na publicação da Resolução ${ }^{\circ}{ }^{89}$, de 16 de março de $2004^{25}$. Ainda, exigiu que os testes de controle de qualidade da droga vegetal e produto acabado fossem realizados por instituições credenciadas à Rede Brasileira de Laboratórios em Saúde (Reblas) (quando terceirizados) ou por empresas fabricantes de medicamentos que tenham certificado de Boas Práticas de Fabricação.

Em 2010, entrou em vigor a Resolução $n^{\circ} 14$ (31 de março de $2010)^{26}$, que contribuiu identificando os fitoterápicos que utilizaram literatura e estudos etnofarmacológicos para comprovação de segurança e eficácia com a seguinte frase no rótulo e bula:
"Medicamento registrado com base no uso tradicional, não sendo recomendado seu uso por período prolongado". Além disso, o registro simplificado de fitoterápicos seguiu publicação da "Lista de Medicamentos Fitoterápicos de Registro Simplificado" por meio da Instrução Normativa $n^{\circ} 5$, de 11 de dezembro de $2008^{27}$ que listou 36 espécies de planta na lista de medicamentos fitoterápicos.

A atual legislação, Resolução nº 26 (13 de maio de 2014) ${ }^{1}$, trouxe um diferencial em relação às outras diretrizes, classificando os fitoterápicos em duas classes de medicamentos: medicamentos fitoterápicos (MF) e produtos tradicionais fitoterápicos (PTF). Os PTF foram classificados dessa forma para permitir a identificação, pela população, dos fitoterápicos que tiveram a sua comprovação de eficácia e segurança realizadas através de literatura técnico-cientifica, daqueles medicamentos que tiveram a sua comprovação através de estudos clínicos (MF). Além disso, incluiu o acompanhamento pós-registro dos fitoterápicos evidenciando a necessidade de notificação no sistema de farmacovigilância, regulamentado pela $\mathrm{RDC} \mathrm{n}^{\circ} 4$, de 13 de maio de 2009 que trouxe em seu escopo o Guia de Farmacovigilância para Detentores de Registro de Medicamentos ${ }^{28}$.

No Brasil, a legislação para medicamentos fitoterápicos vem sofrendo modificações nos últimos vinte anos. Se a primeira legislação que regulamentou os fitoterápicos surgiu em 1967, após o ano de 1995 foram publicados cinco documentos que modificam o registro de fitoterápicos no Brasil: Portaria SVS/MS $\mathrm{n}^{\circ}$ 6, de 31 de janeiro de $1995^{21} ; \mathrm{RDC} \mathrm{n}^{\circ} 17$, de 24 de fevereiro de 200023; RDC $n^{\circ} 48$, de 16 de março de 2004²4 RDC $n^{\circ} 14$, de 31 de março de $2010^{26}$, e a RDC n²6, de 13 de maio de $2014^{1}$, atualmente em vigor, que dispõe sobre o registro de medicamentos fitoterápicos.

A preocupação das autoridades regulatórias com a normatização dos medicamentos em questão propicia a avaliação de aspectos importantes, como a eficácia e segurança do uso destes medicamentos. 0 uso tradicional de diversas plantas medicinais baseado em conhecimentos populares aliado à crença de que, por serem naturais, não causam reações adversas fizeram com que poucas plantas medicinais fossem avaliadas através de estudos pré-clínicos e clínicos, a fim de comprovar sua eficácia e segurança ${ }^{29}$. Além disto, sabe-se que muitas plantas medicinais apresentam substâncias que podem desencadear reações adversas, seja por seus próprios componentes, seja pela presença de contaminantes ou adulterantes presentes nas preparações fitoterápicas, exigindo um rigoroso controle de qualidade desde o cultivo, coleta da planta, extração de seus constituintes até a elaboração do medicamento ${ }^{30,31}$.

\section{DISCUSSÃO}

O conhecimento de plantas medicinais e seu emprego no tratamento de doenças foram possíveis por meio da realização de estudos etnofarmacológicos em várias regiões brasileiras e, em parte, pela comprovação de seus usos através de estudos clínicos. A iniciativa da CIPLAN em implantar a fitoterapia nos serviços primários de saúde foi o primeiro passo para condicionar o uso de plantas medicinais a estudos científicos ${ }^{32}$. 
A RDC $n^{\circ} 26 / 14^{1}$ é o reflexo de como a legislação brasileira se adaptou a essa tendência. Se, em 1995, tínhamos uma legislação para fitoterápicos que se assemelhava com o registro de medicamentos sintéticos, atualmente presenciamos uma legislação que cria mecanismos para flexibilizar a disponibilização de medicamentos fitoterápicos no mercado. 0 registro simplificado e o registro com base em documentação técnica-científica são provas disso. A legislação atual se tornou transparente, pois permitiu a visualização imediata de como a comprovação de segurança e eficácia foi realizada, classificando os medicamentos em MF e PTF. Essa medida só adiciona maior credibilidade e confiança no tratamento medicamentoso com fitoterápicos.

Em um estudo realizado em Pelotas ${ }^{33}$, em que foram entrevistados 393 profissionais de saúde e pacientes em cinco unidades de saúde do SUS, observou-se que $91,6 \%$ dos pacientes e $65,0 \%$ dos profissionais de saúde já utilizaram fitoterápico, pelo menos uma vez, para o tratamento de alguma doença. Entre esses profissionais, porém, foi constatado que somente $10,0 \%$ prescreviam fitoterápicos aos pacientes. Apesar de ser um exemplo isolado, sem dúvida, é um indicativo da baixa adesão dos profissionais de saúde a prescrição do medicamento fitoterápico, muitas vezes pelo descrédito na terapêutica, pela ausência de conhecimento técnico-científico aliado ao fato de poucas universidades possuírem em sua grade curricular disciplinas voltadas ao uso e prescrição de fitoterápicos. Para a população, o uso de fitoterápicos se confunde muitas vezes com o uso de plantas ditas medicinais utilizadas na forma de preparações extrativas (chá e decoctos) as quais não configuram um fitoterápico, pois não possuem identidade botânica segura e estas se correlacionam com a ideia "o que é natural não faz mal".

Dentro desta perspectiva, o Formulário de Fitoterápicos da Farmacopeia Brasileira (FFFB), publicado pela RDC $n^{\circ} 60$, de 10 de novembro de $2011^{34}$, e posteriormente o Memento Fitoterápico (MFFB) ${ }^{35}$, publicado em 2016, pela Resolução $n^{\circ} 84 / 16$, servem como documentos de consulta rápida por profissionais prescritores. Em forma de monografias, os documentos trazem informações referentes às indicações terapêuticas, vias de administração, posologia, interações medicamentosas, formas farmacêuticas, entre outras informações de fitoterápicos que possibilitam o profissional de saúde avaliar de maneira segura a necessidade de prescrição ao paciente.

Nesse sentido, a inserção do FFFB e do MFFB na prática da fitoterapia clínica é de fundamental importância. Aliado à RDC $\mathrm{n}^{\circ}$ $26 / 14^{1}$, servem como ferramentas para viabilizar as prescrições de fitoterápicos no SUS. Se o que se observa nas unidades de saúde é a baixa adesão dos profissionais de saúde à prescrição de fitoterápicos, os documentos em vigor trazem confiança, segurança e estímulo com relação aos medicamentos fitoterápicos e podem refletir positivamente no aumento das prescrições pelos profissionais de saúde do SUS.

$\mathrm{O}$ conjunto de políticas adotadas até o momento, de forma indireta, estimula a pesquisa de plantas medicinais. 0 estudo de novas plantas medicinais e a comprovação de eficácia e segurança é de extrema importância para a inclusão de novas monografias na Farmacopeia Brasileira. A regularidade na atualização da lista de incorporação de fitoterápicos no SUS (Renisus) é mais um instrumento para o aumento das prescrições fitoterápicas, pois reflete as necessidades dos usuários do SUS e por consequência estimula a pesquisa na área de produtos naturais.

Para tanto, é preciso incentivar ações de sensibilização de médicos, enfermeiros, nutricionistas e farmacêuticos para estimular a prescrição dos medicamentos e produtos fitoterápicos, quando disponíveis na unidade de saúde. Além de viabilizar junto às diretrizes curriculares no Brasil que esta área seja contemplada nos currículos, é necessário promover junto aos conselhos de classe cursos de aperfeiçoamento para clínica em fitoterapia. É necessário também incentivar ações que regulamentem a prescrições de fitoterápicos pelos profissionais de saúde. Como exemplo, o Conselho Federal de Nutricionistas, por meio da Resolução CFN n 525 , de 25 de junho de $2013^{36}$, regulamentou a prática da fitoterapia pelo nutricionista, atribuindo-lhe competência para prescrever plantas medicinais, drogas vegetais e fitoterápicos como complemento da prescrição dietética, desde que isentos de prescrição médica. Da mesma forma ocorreu com a publicação da Resolução $n^{\circ} 586$, de 29 de agosto de 2013 pelo Conselho Federal de Farmácia, que regulou a prescrição farmacêutica de fitoterápicos isentos de prescrição médica ${ }^{37}$.

A fitoterapia experimenta um novo patamar com as recentes publicações, trazendo credibilidade e segurança aos pacientes e profissionais da saúde, transformando a fitoterapia não apenas em uma terapia alternativa, mas sim em uma terapia na qual os medicamentos fitoterápicos, em alguns casos, poderão ser considerados como tratamento de primeira escolha.

\section{CONCLUSÕES}

Após dez anos do lançamento da PNPMF, observa-se a publicação de monografias de drogas vegetais cada vez mais detalhadas como forma de definir os critérios para uma utilização segura dos fitoterápicos. $A R D C n^{\circ} 26 / 14^{1}$ concretizou esta tendência reunindo em seu escopo critérios para controle de qualidade, boas práticas de fabricação, validação de métodos analíticos, estabilidade e farmacovigilância. Esta última, uma importante ferramenta na avaliação de eficácia e segurança e fonte de dados para o incentivo à pesquisa e ao aprimoramento das monografias disponibilizadas pela Farmacopeia. Sem dúvida, um desafio a ser aprimorado para garantir uma boa qualidade de vida à população brasileira.

\section{REFERÊNCIAS}

1. Agência Nacional de Vigilância Sanitária - Anvisa. Resolução da Diretoria Colegiada - RDC $n^{\circ} 26$, de 13 de maio de 2014. Dispõe sobre o registro de medicamentos fitoterápicos e o registro e a notificação de produtos tradicionais fitoterápicos. Diário Oficial União. 14 maio 2014; Seção 1:52. 
2. World Health Organization - WHO. Traditional medicine. Geneva: WHO; 1977[acesso 26 maio 2016]. Disponível em: http://apps.who.int/iris/bitstream/10665/12727 1/2/ sea_racmr_77.1_10.pdf

3. World Health Organization - WHO. WHA 30.49 Promotion and development of training and research in traditional medicine. In: 30th. World Healtyh Assembly; 2-19 May 1977[acesso 26 maio 2016]; Geneva. Disponível em: http: // www.who.int/medicines/areas/traditional/wha3049.pdf

4. Declaração de Alma-Ata. In: 4a Conferência Internacional Sobre Cuidados Primários de Saúde; 1978[acesso 26 maio 2016]. Alma Ata, URSS. Disponível em: http:// cmdss2011.org/site/wpcontent/uploads/2011/07/ Declara\%C3\%A7\%C3\%A3o-Alma-Ata.pdf

5. World Health Organization - WHO. WHA 31.33 Medicinal plants. Geneva: World Health Organization; 1978[acesso 25 maio 2016]. Disponível em: http://www.who.int/ medicines/areas/traditional/wha3133.pdf

6. World Health Organization - WHO. WHA 40.33 Traditional medicine. Geneva: World Health Organization; 1987[acesso 25 maio 2016]. Disponível em: http://www.searo.who.int/ entity /medicines/topics/wha4033.pdf

7. World Health Organization - WHO. General guidelines for methodologies on research and evaluation of traditional medicine. Geneva: World Health Organization; 2000.

8. World Health Organization - WHO. WHO Traditional medicine strategy: 2014-2023. Geneva: World Health Organization; 2013.

9. Ministério da Saúde (BR). Portaria $n^{\circ} 971$, de 3 de maio de 2006. Aprova a Política Nacional de Práticas Integrativas e Complementares (PNPIC) no Sistema Único de Saúde. Diário Oficial União. 4 maio 2006.

10. Brasil.Comissão Interministerial de Planejamento e Coordenação - CIPLAN. Resolução ${ }^{\circ} 8$, de 08 de março de 1988. Criar procedimentos e implantar técnicas alternativas de saúde mental nos serviços médico-psicológicoassistenciais e áreas afins. Diário Oficial União. 11 mar 1988.

11. Ministério da Saúde (BR). Relatório da $10^{\mathrm{a}}$ Conferência Nacional de Saúde. Brasília, DF: Ministério da Saúde; 1996.

12. Ministério da Saúde (BR). Portaria $n^{\circ} 3.916$, de 30 de outubro de 1998. Aprovar a Política Nacional de Medicamentos. Diário Oficial União. 10 nov 1998;Seção 1:18-22.

13. Seminário Nacional de Plantas Medicinais, Fitoterápicos e Assistência Farmacêutica: preparatório à Conferência Nacional de Medicamentos e Assistência Farmacêutica. Brasília, DF: Ministério da Saúde; 2013.

14. Conferência Nacional de Saúde. $12^{\text {a }}$ Conferência Nacional de Saúde: Conferência Sergio Arouca: Brasília, 7 a 11 de dezembro de 2003: relatório final. Brasília: Ministério da Saúde, 2004.

15. Conselho Nacional de Saúde. Resolução n 338 , de 6 de maio de 2004. Aprovar a Política Nacional de Assistência Farmacêutica. Diário Oficial União. 20 maio 2004;Seção 1:52.

16. Brasil. Decreto $n^{\circ} 5.813$, de 22 de junho de 2006. Aprova a Política Nacional de Plantas Medicinais e Fitoterápicos e dá outras providências. Diário Oficial União. 23 jun 2006.
17. Agência Saúde. MS elabora relação de plantas medicinais de interesse ao SUS. Brasília, DF: Ministério da Saúde; 2009[acesso 25 maio 2016]. Disponível em: http: / /bvsms. saude.gov.br/bvs/sus/pdf/marco/ms_relacao_plantas_medi cinais_sus_0603.pdf

18. Portal da Saúde. Plantas de interesse ao SUS. Brasília, DF: Ministério da Saúde; 2014[acesso 26 maio 2016] Disponível em: http://portalsaude.saude.gov.br/index.php/oministerio /principal/leia-mais-o-ministerio/465-sctie-raiz/ daf-raiz/ceaf-sctie/fitoterapicos-cgafb/l1-fitoterapicos /12552-plantas-de-interesse-ao-sus

19. Torres KR. Política Nacional de Plantas Medicinais e Fitoterápicos. In: 3. EnFarMed, set. 2009, São Paulo. Brasília, DF: Miistério da Saúde; 2009.

20. Brasil. Portaria $n^{\circ} 22$, de 30 de outubro de 1967. Estabelece normas para o emprego de preparações fitoterápicas. Diário Oficial União. 16 nov 1967.

21. Brasil. Portaria $n^{\circ} 6$, de 31 de janeiro de 1995. Instituir e normatizar o registro de produtos fitoterápicos junto ao Sistema de Vigilância Sanitária. Diário Oficial União. $6 \mathrm{fev}$ 1995;Seção 1:1523.

22. Brasil. Lei $n^{\circ} 6360$, de 23 de setembro de 1976. Dispõe sobre a vigilância sanitária a que ficam sujeitos os medicamentos, as drogas, os insumos farmacêuticos e correlatos, cosméticos, saneantes e outros produtos, e dá outras Providências. Diário Oficial União. 24 set 1976.

23. Agência Nacional de Vigilância Sanitária - Anvisa. Resolução $\mathrm{RDC} \mathrm{n}^{\circ}$ 17, de 24 de fevereiro de 2000. Dispõe sobre o registro de medicamento fitoterápico. Diário Oficial União. 24 abr 2000; Seção 1:25.

24. Agência Nacional de Vigilância Sanitária - Anvisa. Resolução $\mathrm{RDC} \mathrm{n}^{\circ} 48$, de 16 de março de 2004. Dispõe sobre o registro de medicamento fitoterápico. Diário Oficial União. $18 \mathrm{mar}$ 2004;Seção 1:39.

25. Agência Nacional de Vigilância Sanitária - Anvisa. Resolução RDC $n^{\circ} 89$, de 16 de março de 2004. Resolução $n^{\circ} 89$, de 16 de março de 2004. Determina a publicação da Lista de Registro Simplificado de Fitoterápicos. Diário Oficial União. 18 mar 2004;Seção 1:32.

26. Agência Nacional de Vigilância Sanitária - Anvisa. Resolução RDC $n^{\circ}$ 14, de 31 de março de 2010. Dispõe sobre o registro de medicamentos fitoterápicos. Diário Oficial União. 1 abr 2010.

27. Agência Nacional de Vigilância Sanitária - Anvisa. Instrução Normativa $n^{\circ} 5$, de 11 de dezembro de 2008. Determina a publicação da "Lista de medicamentos fitoterápicos de registro simplificado" e a "Lista de produtos tradicionais fitoterápicos de registro simplificado”. Diário Oficial União. 12 dez 2008.

28. Agência Nacional de Vigilância Sanitária - Anvisa. Resolução RDC $n^{\circ} 4$, de 10 de fevereiro de 2009. Dispõe sobre as normas de farmacovigilância para os detentores de registro de medicamentos de uso humano. Diário Oficial União.11 fev 2009;Seção 1:42.

29. De Smet P. Health risks of herbal remedies: an update. Clin Pharmacol Ther. 2004;76(1):1-17. doi:10.1016/j.clpt.2004.03.005 
30. Ernst E. Risks of herbal medicinal products. Pharmacoepidemiol Drug Saf. 2004;13(11):767-71. doi:10.1002/pds.1014

31. Hussain K, Majeed MT, Ismail Z, Sadikun A, Ibrahim P. Traditional and complementary medicines: quality assessment startegies and safe usage. South Med Rev. 2009;2(1):19-23.

32. Oliveira AB, Longhi JG, Andrade CA, Miguel OG, Miguel MD. A normatização dos fitoterápicos no Brasil. Visão Acad. 2006;7(2). doi:10.5380/acd.v7i2.9042

33. Oliveira SGD, Moura FRR, Demarco FF, Nascente PS, Pino FA, Lund RG. An ethnomedicinal survey on phytotherapy with professionals and patients from Basic Care Units in the Brazilian Unified Health System. J Ethnopharmacol. 2012;140(2):428-37. doi:10.1016/j.jep.2012.01.054

34. Agência Nacional de Vigilância Sanitária - Anvisa. Resolução $\mathrm{RDC} \mathrm{n}^{\circ} 60$, de 10 de novembro de 2011. Aprova o Formulário de Fitoterápicos da Farmacopéia Brasileira, primeira edição e dá outras providências. Diário Oficial União. 11 nov 2011.

35. Agência Nacional de Vigilância Sanitária - Anvisa. Memento fitoterápico: farmacopéia brasileira. Brasília, DF: Agência Nacional de Vigilância Sanitária; 2016.

36. Conselho Federal de Nutricionistas. Resolução CFN $n^{\circ} 525 / 2013$. Regulamenta a prática da fitoterapia pelo nutricionista, atribuindo-lhe competência para, nas modalidades que especifica, prescrever plantas medicinais, drogas vegetais e fitoterápicos como complemento da prescrição dietética e, dá outras providências.Brasília, DF: Conselho Federal de Nutricionistas; 2013 [acesso 25 maio 2016]. Disponível em: http://www.cfn.org.br/eficiente/ repositorio/legislacao/resolucoes/583.pdf

37. Conselho Federal de Farmácia. Resolução n ${ }^{\circ} 586$, de 29 de agosto de 2013. Regulamenta a prescrição farmacêutca e dá outras providências. Diário Oficial União. 26 set 2013;Seção 1:136-8. 